\title{
SINGULAR PERTURBATIONS OF INTEGRODIFFERENTIAL EQUATIONS IN BANACH SPACE
}

\author{
JAMES H. LIU
}

(Communicated by Palle E. T. Jorgensen)

$$
\begin{aligned}
& \text { Abstract. Let } \varepsilon>0 \text { and consider } \\
& \begin{aligned}
\varepsilon^{2} u^{\prime \prime}(t ; \varepsilon)+u^{\prime}(t ; \varepsilon)= & A u(t ; \varepsilon)+\int_{0}^{t} K(t-s) A u(s ; \varepsilon) d s+f(t ; \varepsilon), \quad t \geq 0, \\
& u(0 ; \varepsilon)=u_{0}(\varepsilon), \quad u^{\prime}(0 ; \varepsilon)=u_{1}(\varepsilon),
\end{aligned}
\end{aligned}
$$

and

$$
w^{\prime}(t)=A w(t)+\int_{0}^{t} K(t-s) A w(s) d s+f(t) \quad t \geq 0, w(0)=w_{0},
$$

in a Banach space $X$ when $\varepsilon \rightarrow 0$. Here $A$ is the generator of a strongly continuous cosine family and a strongly continuous semigroup, and $K(t)$ is a bounded linear operator for $t \geq 0$. With some convergence conditions on initial data and $f(t ; \varepsilon)$ and smoothness conditions on $K(\cdot)$, we prove that if $\varepsilon \rightarrow 0$, then $u(t ; \varepsilon) \rightarrow w(t)$ in $X$ uniformly for $t \in[0, T]$ for any fixed $T>0$. We will apply this to an equation in viscoelasticity.

\section{INTRODUCTION}

We study integrodifferential equations

$$
\begin{aligned}
\varepsilon^{2} u^{\prime \prime}(t ; \varepsilon)+u^{\prime}(t ; \varepsilon)= & A u(t ; \varepsilon)+\int_{0}^{t} K(t-s) A u(s ; \varepsilon) d s \\
& +f(t ; \varepsilon), \quad t \geq 0, \\
u(0 ; \varepsilon)= & u_{0}(\varepsilon), \quad u^{\prime}(0 ; \varepsilon)=u_{1}(\varepsilon),
\end{aligned}
$$

and

$$
\begin{gathered}
w^{\prime}(t)=A w(t)+\int_{0}^{t} K(t-s) A w(s) d s+f(t), \quad t \geq 0 \\
w(0)=w_{0},
\end{gathered}
$$

in a Banach space $X$, with $A$ the generator of a strongly continuous cosine family and a strongly continuous semigroup, and $K(t)$ a bounded linear operator for $t \geq 0$. We regard (1.2) as the limiting equation of (1.1) as $\varepsilon \rightarrow 0$.

Received by the editors July 5, 1991 and, in revised form, February 22, 1993.

1991 Mathematics Subject Classification. Primary 47J05, 45N05, 47Dxx.

Key words and phrases. Singular perturbation, convergence in solutions. 
Now, (1.2) is of lower order of derivative (in $t$ ); in this sense we say that we are dealing with the singular perturbation problems.

There are many studies on singular perturbations (see, e.g., Goldstein [6], Hale and Raugel [10], Smith [13], Grimmer and Liu [8], and the references therein). Since this work was influenced by Fattorini [5], we only state some results of [5].

Fattorini [5] considered the singular perturbations for

$$
\begin{gathered}
\varepsilon^{2} u^{\prime \prime}(t ; \varepsilon)+u^{\prime}(t ; \varepsilon)=A u(t ; \varepsilon)+f(t ; \varepsilon), \quad t \geq 0 \\
u(0 ; \varepsilon)=u_{0}(\varepsilon), \quad u^{\prime}(0 ; \varepsilon)=u_{1}(\varepsilon),
\end{gathered}
$$

and

$$
w^{\prime}(t)=A w(t)+f(t), \quad t \geq 0, \quad w(0)=w_{0},
$$

with $A$ the generator of a strongly continuous cosine family and a strongly continuous semigroup in a Banach space $X$ and proved that:

For any $T>0$, if $f(\cdot ; \varepsilon) \rightarrow f$ in $L^{1}([0, T], X)$ and $u_{0}(\varepsilon) \rightarrow$ $w_{0}, \varepsilon^{2} u_{1}(\varepsilon) \rightarrow 0$ as $\varepsilon \rightarrow 0$, then $u(t ; \varepsilon) \rightarrow w(t)$ in $X$ uniformly for $t \in[0, T]$ as $\varepsilon \rightarrow 0$.

We will prove here with some smoothness conditions on $K(\cdot)$ that exactly the same statements as above hold for (1.1) and (1.2). The methods we will use in studying the singular perturbations for integrodifferential equations are as follows: We first use the technique introduced in $[1,2,11,12]$ to change (1.1) and (1.2) into equations that look like (1.3) and (1.4), and then estimate $u(t ; \varepsilon)-w(t)$. Note that $u(\cdot ; \varepsilon)-w(\cdot)$ will also appear as an integrand, so Gronwall's inequality is used to solve the problem. Finally we apply this result to an equation in viscoelasticity.

\section{Singular PERTURbations}

In this paper we make the following hypotheses:

(H1) Operator $A$ generates a strongly continuous cosine family $C(\cdot)$ and a strongly continuous semigroup $S(\cdot)$. (See [5].)

(H2) For $t \geq 0, K(t), K^{\prime}(t), K^{\prime \prime}(t) \in B(X)(B(X)=$ space of all bounded linear operators on $X)$. For $x \in X, K x, K^{\prime} x, K^{\prime \prime} x \in L_{\text {loc }}^{1}\left(R^{+}, X\right)$. Here $K^{\prime}, K^{\prime \prime}$ are the strong derivatives.

(H3) $f(\cdot ; \varepsilon), f \in C^{1}\left(R^{+}, X\right)$, where $\varepsilon>0, R^{+}=[0, \infty)$.

We say that $u: R^{+} \rightarrow X$ is a solution of $(1.1)$ if $u \in C^{2}\left(R^{+}, X\right), u(t) \in D(A)$ (domain of $A$ ) for $t \geq 0$ and (1.1) is satisfied on $R^{+}$. Solutions of (1.2) are defined in a similar way. In order to verify the existence of solutions of (1.1) we change it to another more common form. (See [5].) Let

$$
u(t ; \varepsilon)=e^{-t / 2 \varepsilon^{2}} v(t / \varepsilon)
$$

Then (1.1) can be replaced by

$$
v^{\prime \prime}(t / \varepsilon)=\left(A+\frac{1}{4 \varepsilon^{2}}\right) v(t / \varepsilon)+\int_{0}^{t} K(t-s) e^{(t-s) / 2 \varepsilon^{2}} A v(s / \varepsilon) d s+e^{t / 2 \varepsilon^{2}} f(t ; \varepsilon)
$$


Now let $h=t / \varepsilon$, and then change $h$ to $t$ to get

$$
\begin{gathered}
v^{\prime \prime}(t)=\left(A+\frac{1}{4 \varepsilon^{2}}\right) v(t)+\int_{0}^{t} \hat{K}(t-s) A v(s) d s+\hat{f}(t), \\
v(0 ; \varepsilon)=u_{0}(\varepsilon), \quad v^{\prime}(0 ; \varepsilon)=\frac{1}{2 \varepsilon} u_{0}(\varepsilon)+\varepsilon u_{1}(\varepsilon)
\end{gathered}
$$

where $\left(A+\frac{1}{4 \varepsilon^{2}}\right)$ generates a strongly continuous cosine family and

$$
\hat{K}(t)=\varepsilon K(\varepsilon t) e^{t / 2 \varepsilon}, \quad \hat{f}(t)=f(\varepsilon t ; \varepsilon) e^{t / 2 \varepsilon}, \quad t \geq 0 .
$$

Note that the existence and uniqueness of solutions of (2.1) and (1.2) were obtained in $[3,4,7,14,15]$ and that we are only interested in singular perturbations in this paper. Thus, we may assume that (1.1) and (1.2) have unique solutions $u(t ; \varepsilon)$ and $w(t)$ respectively for every $\varepsilon>0$.

Now we can state and prove the following result concerning the convergence of solutions, with the following hypotheses:

(H4) $u_{0}(\varepsilon), w_{0} \in D(A), u_{0}(\varepsilon) \rightarrow w_{0}, \varepsilon^{2} u_{1}(\varepsilon) \rightarrow 0$, as $\varepsilon \rightarrow 0$.

(H5) For any $T>0, f(\cdot ; \varepsilon) \rightarrow f(\cdot)$ in $L^{1}([0, T], X)$ as $\varepsilon \rightarrow 0$.

Theorem 2.1. Assume that hypotheses (H1)-(H5) are satisfied. Then for any $T>0, u(t ; \varepsilon) \rightarrow w(t)$ in $X$ uniformly for $t \in[0, T]$, as $\varepsilon \rightarrow 0$.

Proof. Define

$$
R * H(t)=\int_{0}^{t} R(t-s) H(s) d s \text { and } \delta * H=H
$$

Then we can find the solution $F$ of $F+K+F * K=0$ (see $[1,2,11,12])$ so that

$$
(\delta+F) *(\delta+K)=\delta
$$

Now write (1.1) as

$$
\varepsilon^{2} u^{\prime \prime}(\varepsilon)+u^{\prime}(\varepsilon)=(\delta+K) * A u(\varepsilon)+f(\varepsilon) .
$$

Then we have

$$
(\delta+F) *\left[\varepsilon^{2} u^{\prime \prime}(\varepsilon)+u^{\prime}(\varepsilon)\right]=A u(\varepsilon)+(\delta+F) * f(\varepsilon) .
$$

Hence,

$$
\varepsilon^{2} u^{\prime \prime}(\varepsilon)+u^{\prime}(\varepsilon)=A u(\varepsilon)+(\delta+F) * f(\varepsilon)-F *\left[\varepsilon^{2} u^{\prime \prime}(\varepsilon)+u^{\prime}(\varepsilon)\right] .
$$

Integration by parts yields

$$
\begin{aligned}
F * u^{\prime}(t ; \varepsilon)= & \int_{0}^{t} F^{\prime}(t-s) u(s ; \varepsilon) d s+F(0) u(t ; \varepsilon)-F(t) u_{0}(\varepsilon) \\
F * u^{\prime \prime}(t ; \varepsilon)= & \int_{0}^{t} F^{\prime \prime}(t-s) u(s ; \varepsilon) d s+F(0) u^{\prime}(t ; \varepsilon)-F(t) u_{1}(\varepsilon) \\
& +F^{\prime}(0) u(t ; \varepsilon)-F^{\prime}(t) u_{0}(\varepsilon)
\end{aligned}
$$


Therefore (1.1) can be replaced by

$$
\begin{gathered}
\varepsilon^{2} u^{\prime \prime}(t ; \varepsilon)+u^{\prime}(t ; \varepsilon)=A u(t ; \varepsilon)+\hat{f}(t ; \varepsilon), \quad t \geq 0 \\
u(0 ; \varepsilon)=u_{0}(\varepsilon), \quad u^{\prime}(0 ; \varepsilon)=u_{1}(\varepsilon)
\end{gathered}
$$

with

$$
\begin{aligned}
\hat{f}(t ; \varepsilon)= & (\delta+F) * f(t ; \varepsilon)-F *\left[\varepsilon^{2} u^{\prime \prime}(t ; \varepsilon)+u^{\prime}(t ; \varepsilon)\right] \\
= & (\delta+F) * f(t ; \varepsilon)-\int_{0}^{t} F^{\prime}(t-s) u(s ; \varepsilon) d s-F(0) u(t ; \varepsilon)+F(t) u_{0}(\varepsilon) \\
& -\varepsilon^{2}\left[\int_{0}^{t} F^{\prime \prime}(t-s) u(s ; \varepsilon) d s+F^{\prime}(0) u(t ; \varepsilon)\right. \\
& \left.-F^{\prime}(t) u_{0}(\varepsilon)+F(0) u^{\prime}(t ; \varepsilon)-F(t) u_{1}(\varepsilon)\right]
\end{aligned}
$$

Similarly, (1.2) can be replaced by

$$
w^{\prime}(t)=A w(t)+\hat{f}(t), \quad t \geq 0, \quad w(0)=w_{0},
$$

with

$$
\begin{aligned}
\hat{f}(t) & =(\delta+F) * f(t)-F * w^{\prime}(t) \\
& =(\delta+F) * f(t)-\int_{0}^{t} F^{\prime}(t-s) w(s) d s-F(0) w(t)+F(t) w_{0}
\end{aligned}
$$

So we can use results in [5] to get for $t \geq 0$

$$
\begin{aligned}
w(t)= & S(t) w_{0}+\int_{0}^{t} S(t-s) \hat{f}(s) d s \\
u(t ; \varepsilon)= & e^{-t / 2 \varepsilon^{2}} C(t / \varepsilon) u_{0}(\varepsilon)+\frac{1}{2} R(t, \varepsilon) u_{0}(\varepsilon) \\
& +G(t, \varepsilon)\left[\frac{1}{2} u_{0}(\varepsilon)+\varepsilon^{2} u_{1}(\varepsilon)\right]+\int_{0}^{t} G(t-s) \hat{f}(s, \varepsilon) d s,
\end{aligned}
$$

where $S(\cdot), C(\cdot)$ are given in $(\mathrm{H} 1), R(\cdot ; \varepsilon), G(\cdot ; \varepsilon)$ are linear operators defined in [5] using the Bessel functions, and they have the following properties: For some constants $\alpha, \omega>0$,

(P1) $\|C(t)\|,\|S(t)\| \leq \alpha e^{\omega^{2} t}, t \geq 0, \varepsilon>0$.

(P2) $\|G(t ; \varepsilon)\|, \varepsilon^{2}\left\|G^{\prime}(t ; \varepsilon)\right\| \leq \alpha e^{\omega^{2} t}, t \geq 0, \varepsilon>0$.

(P3) $\varepsilon^{2} G^{\prime}(t ; \varepsilon)=e^{-t / 2 \varepsilon^{2}} C(t / \varepsilon)+\frac{1}{2}[R(t ; \varepsilon)-G(t ; \varepsilon)]$. $T>0$,

(P4) If $t(\varepsilon)>0$ for $\varepsilon>0$ with $t(\varepsilon) / \varepsilon^{2} \rightarrow \infty$ as $\varepsilon \rightarrow 0$, then for every $\lim _{\varepsilon \rightarrow 0} \sup _{t(\varepsilon) \leq t \leq T}\|R(t ; \varepsilon) x-S(t) x\|=0 \quad$ and $\quad \lim _{\varepsilon \rightarrow 0} \sup _{t(\varepsilon) \leq t \leq T}\|G(t ; \varepsilon) x-S(t) x\|=0$, uniformly for $x$ in bounded subsets of $X$. 
(P5)

$$
\begin{aligned}
& \| e^{-t / 2 \varepsilon^{2}} C(t / \varepsilon) u_{0}(\varepsilon)+\frac{1}{2} R(t ; \varepsilon) u_{0}(\varepsilon) \\
& \quad+G(t ; \varepsilon)\left[\frac{1}{2} u_{0}(\varepsilon)+\varepsilon^{2} u_{1}(\varepsilon)\right]-S(t) w_{0} \| \\
& \quad \leq \alpha e^{\omega^{2} t}\left[\varepsilon^{2}\left(1+\omega^{2} t\right)\left\|A w_{0}\right\|+\left\|u_{0}(\varepsilon)-w_{0}\right\|+\varepsilon^{2}\left\|u_{1}(\varepsilon)\right\|\right], \quad t \geq 0 .
\end{aligned}
$$

Now let $T>0$ be fixed and consider for $t \in[0, T]$,

$$
\begin{aligned}
u(t ; \varepsilon)-w(t)= & e^{-t / 2 \varepsilon^{2}} C(t / \varepsilon) u_{0}(\varepsilon)+\frac{1}{2} R(t ; \varepsilon) u_{0}(\varepsilon) \\
& +G(t ; \varepsilon)\left[\frac{1}{2} u_{0}(\varepsilon)+\varepsilon^{2} u_{1}(\varepsilon)\right] \\
& -S(t) w_{0}+\int_{0}^{t}[G(t-s ; \varepsilon) \hat{f}(s ; \varepsilon)-S(t-s) \hat{f}(s)] d s
\end{aligned}
$$

By (H4) and (P5), we can write (2.7) as

$$
\begin{aligned}
u(t ; \varepsilon)-w(t)= & 0(\varepsilon,[0, T])+\int_{0}^{t}[G(t-s ; \varepsilon) \hat{f}(s ; \varepsilon)-S(t-s) \hat{f}(s)] d s \\
= & 0(\varepsilon,[0, T])+\int_{0}^{t} G(t-s ; \varepsilon)[\hat{f}(s ; \varepsilon)-\hat{f}(s)] d s \\
& +\int_{0}^{t}[G(t-s ; \varepsilon)-S(t-s)] \hat{f}(s) d s,
\end{aligned}
$$

where $0(\varepsilon,[0, T])$ satisfies

$$
0(\varepsilon,[0, T]) \rightarrow 0 \text { as } \varepsilon \rightarrow 0 \text {, uniformly for } t \in[0, T] .
$$

Note that $w$ is locally bounded and $f \in L^{1}([0, T], X)$; then $\hat{f} \in L^{1}([0, T], X)$. So from [5],

$$
\int_{0}^{t}[G(t-s ; \varepsilon)-S(t-s)] \hat{f}(s) d s=0(\varepsilon,[0, T]), \quad t \in[0, T]
$$

Next, we have

$$
\begin{aligned}
\int_{0}^{t} G(t-s ; \varepsilon)[\hat{f}(s ; \varepsilon)-\hat{f}(s)] d s \\
=\int_{0}^{t} G(t-s ; \varepsilon)\left[\hat{f}(s ; \varepsilon)-\hat{f}(s)+\varepsilon^{2} F(0) u^{\prime}(s ; \varepsilon)\right] d s \\
\quad-\int_{0}^{t} G(t-s ; \varepsilon) \varepsilon^{2} F(0) u^{\prime}(s ; \varepsilon) d s .
\end{aligned}
$$


Now from (P3),

$$
\begin{aligned}
\int_{0}^{t} G( & -s ; \varepsilon) \varepsilon^{2} F(0) u^{\prime}(s ; \varepsilon) d s \\
= & \varepsilon^{2} G(0 ; \varepsilon) F(0) u(t ; \varepsilon)-\varepsilon^{2} G(t ; \varepsilon) F(0) u_{0}(\varepsilon) \\
& +\varepsilon^{2} \int_{0}^{t} G^{\prime}(t-s ; \varepsilon) F(0) u(s ; \varepsilon) d s \\
= & \varepsilon^{2} G(0 ; \varepsilon) F(0) u(t ; \varepsilon)-\varepsilon^{2} G(t ; \varepsilon) F(0) u_{0}(\varepsilon) \\
& +\varepsilon^{2} \int_{0}^{t} G^{\prime}(t-s ; \varepsilon) F(0)[u(s ; \varepsilon)-w(s)] d s \\
& +\varepsilon^{2} \int_{0}^{t} G^{\prime}(t-s ; \varepsilon) F(0) w(s) d s \\
= & \varepsilon^{2} G(0 ; \varepsilon) F(0)[u(t ; \varepsilon)-w(t)]+\varepsilon^{2} G(0 ; \varepsilon) F(0) w(t) \\
& -\varepsilon^{2} G(t ; \varepsilon) F(0) u_{0}(\varepsilon) \\
& +\varepsilon^{2} \int_{0}^{t} G^{\prime}(t-s ; \varepsilon) F(0)[u(s ; \varepsilon)-w(s)] d s \\
& +\int_{0}^{t}\left\{e^{-(t-s) / 2 \varepsilon^{2}} C((t-s) / \varepsilon)\right. \\
& \left.+\frac{1}{2}[R(t-s ; \varepsilon)-G(t-s ; \varepsilon)]\right\} F(0) w(s) d s .
\end{aligned}
$$

Observe that $w(s)$ is locally bounded, so use property (P4) with $t(\varepsilon)=\varepsilon$ to obtain for any $t, s \in[0, T]$ with $s<t$,

$$
[R(t-s ; \varepsilon)-G(t-s ; \varepsilon)] F(0) w(s) \rightarrow 0, \quad \varepsilon \rightarrow 0
$$

Hence, the dominated convergence theorem can be used to prove that

$$
\int_{0}^{t}[R(t-s ; \varepsilon)-G(t-s ; \varepsilon)] F(0) w(s) d s \rightarrow 0, \quad \varepsilon \rightarrow 0
$$

uniformly for $t \in[0, T]$. Next, assume that $\varepsilon>0$ is so small that $4 \varepsilon \omega^{2} \leq 1$. Then from (P1)

$$
\begin{aligned}
& \int_{0}^{t} e^{-(t-s) / 2 \varepsilon^{2}}\|C((t-s) / \varepsilon)\| d s=\int_{0}^{t} e^{-s / 2 \varepsilon^{2}}\|C(s / \varepsilon)\| d s \\
& \quad \leq \alpha \int_{0}^{t} e^{-s / 2 \varepsilon^{2}+\omega^{2} s / \varepsilon} d s=\left[2 \alpha \varepsilon^{2} /\left(1-2 \varepsilon \omega^{2}\right)\right]\left[1-e^{\left(2 \varepsilon \omega^{2}-1\right) t / 2 \varepsilon^{2}}\right] \\
& \quad \leq 4 \alpha \varepsilon^{2} \rightarrow 0, \quad \varepsilon \rightarrow 0,
\end{aligned}
$$


uniformly for $t \in[0, T]$. Also observe that $w(\cdot)$ is locally bounded and $u_{0}(\varepsilon)$ has a limit as $\varepsilon \rightarrow 0$. Then from (P2)

$$
\varepsilon^{2} G(0 ; \varepsilon) F(0) w(t), \quad \varepsilon^{2} G(t ; \varepsilon) F(0) u_{0}(\varepsilon) \longrightarrow 0, \quad \varepsilon \rightarrow 0,
$$

uniformly for $t \in[0, T]$, and

$$
\begin{array}{r}
\left\|\varepsilon^{2} \int_{0}^{t} G^{\prime}(t-s ; \varepsilon) F(0)[u(s ; \varepsilon)-w(s)] d s\right\| \\
\leq \alpha e^{\omega^{2} T}\|F(0)\| \int_{0}^{t}\|u(s ; \varepsilon)-w(s)\| d s .
\end{array}
$$

Thus by (2.12), (2.14), (2.15), and property (P2) we obtain

$$
\begin{aligned}
& \left\|\int_{0}^{t} G(t-s ; \varepsilon) \varepsilon^{2} F(0) u^{\prime}(s ; \varepsilon) d s-\varepsilon^{2} G(0 ; \varepsilon) F(0)[u(t ; \varepsilon)-w(t)]\right\| \\
& \quad \leq(\text { type } 1)+0(\varepsilon,[0, T])
\end{aligned}
$$

where (type 1) is of the form

$$
\text { (const) } \int_{0}^{t}\|u(s ; \varepsilon)-w(s)\| d s
$$

Next we have

$$
\begin{aligned}
\int_{0}^{t} G(t-s ; \varepsilon)[\hat{f}(s ; \varepsilon) & \left.-\hat{f}(s)+\varepsilon^{2} F(0) u^{\prime}(s ; \varepsilon)\right] d s \\
=\int_{0}^{t} G(t-s ; \varepsilon)\{[ & f(s ; \varepsilon)-f(s)]+\int_{0}^{s} F(s-h)[f(h ; \varepsilon)-f(h)] d h \\
& +F(s)\left[u_{0}(\varepsilon)-w_{0}\right]-\int_{0}^{s} F^{\prime}(s-h)[u(h ; \varepsilon)-w(h)] d h \\
& +F(s) \varepsilon^{2} u_{1}(\varepsilon)+\varepsilon^{2} F^{\prime}(s) u_{0}(\varepsilon) \\
& -\left[\varepsilon^{2} F^{\prime}(0)+F(0)\right][u(s ; \varepsilon)-w(s)] \\
& -\varepsilon^{2} F^{\prime}(0) w(s)-\varepsilon^{2} \int_{0}^{s} F^{\prime \prime}(s-h)[u(h ; \varepsilon)-w(h)] d h \\
& \left.\quad-\varepsilon^{2} \int_{0}^{s} F^{\prime \prime}(s-h) w(h) d h\right\} d s
\end{aligned}
$$


Note that from (P2)

$$
\begin{aligned}
& \left\|\int_{0}^{t} G(t-s ; \varepsilon) \int_{0}^{s} F(s-h)[f(h ; \varepsilon)-f(h)] d h d s\right\| \\
& \leq \alpha e^{\omega^{2} T}\left[\int_{0}^{T}\|F(s)\| d s\right]\left[\int_{0}^{T}\|f(s ; \varepsilon)-f(s)\| d s\right], \\
& \left\|\int_{0}^{t} G(t-s ; \varepsilon) F(s)\left[u_{0}(\varepsilon)-w_{0}\right] d s\right\| \\
& \leq \alpha e^{\omega^{2} T}\left\|u_{0}(\varepsilon)-w_{0}\right\| \int_{0}^{T}\|F(s)\| d s, \\
& \left\|\int_{0}^{t} G(t-s ; \varepsilon) \int_{0}^{s} F^{\prime}(s-h)[u(h ; \varepsilon)-w(h)] d h d s\right\| \\
& \leq \alpha e^{\omega^{2} T}\left[\int_{0}^{T}\left\|F^{\prime}(s)\right\| d s\right]\left[\int_{0}^{T}\|u(s ; \varepsilon)-w(s)\| d s\right] .
\end{aligned}
$$

Other terms can be treated similarly. So it is clear that, with property (P2), hypotheses $(\mathrm{H} 1)-(\mathrm{H} 5)$, and the fact that $w(\cdot)$ is locally bounded, we obtain

$$
\begin{aligned}
& \left\|\int_{0}^{t} G(t-s ; \varepsilon)\left[\hat{f}(s ; \varepsilon)-\hat{f}(s)+\varepsilon^{2} F(0) u^{\prime}(s ; \varepsilon)\right] d s\right\| \\
& \quad \leq(\text { type } 1)+0(\varepsilon,[0, T]) .
\end{aligned}
$$

Combining (2.8), (2.10), (2.11), (2.16), and (2.18), we get

$$
\left\|\left(1+\varepsilon^{2} G(0 ; \varepsilon) F(0)\right)[u(t ; \varepsilon)-w(t)]\right\| \leq(\text { type } 1)+0(\varepsilon,[0, T]) .
$$

Now assume $\varepsilon>0$ is so small that $2\left\|\varepsilon^{2} G(0 ; \varepsilon) F(0)\right\|<1$; then

$$
\begin{aligned}
& \|u(t ; \varepsilon)-w(t)\| \\
& \quad \leq 0(\varepsilon,[0, T])+(\text { const }) \int_{0}^{t}\|u(s ; \varepsilon)-w(s)\| d s, \quad t \in[0, T] .
\end{aligned}
$$

So that the Gronwall's inequality [9] can be used to obtain

$$
\|u(t ; \varepsilon)-w(t)\| \leq 0(\varepsilon,[0, T]), \quad t \in[0, T] .
$$

This proves the theorem.

Finally, we briefly indicate its applications in viscoelasticity. Let us consider

$$
\begin{aligned}
\rho u_{t t}(t ; \rho)+\alpha u_{t}(t ; \rho)= & \Delta u(t ; \rho)+\int_{0}^{t} K(t-s) \Delta u(s ; \rho) d s \\
& +f(t ; \rho), \quad t \geq 0, \\
u(0 ; \rho)= & u_{0}(\rho), \quad u_{t}(0 ; \rho)=u_{1}(\rho)
\end{aligned}
$$

in $L^{2}(\Omega)$, where $\mathrm{u}$ is the displacement, $\rho$ is the density per unit area, and $\alpha$ is the coefficient of viscosity of the medium. With appropriate boundary conditions the Laplacian operator $\Delta$ in (2.22) generates a strongly continuous cosine family and a strongly continuous semigroup. So with some convergence 
conditions on initial data and $f(t ; \varepsilon)$ and smoothness conditions on $K(\cdot)$, Theorems 2.1 can be used to show that when density $\rho \rightarrow 0$, solutions of (2.22) will converge to solutions of the "limiting" heat equation

$$
\begin{gathered}
\alpha w_{t}(t)=\Delta w(t)+\int_{0}^{t} K(t-s) \Delta w(s) d s+f(t), \quad t \geq 0, \\
w(0)=w_{0} .
\end{gathered}
$$

Details are omitted here. This result also relates to a concept called "change the type" (from hyperbolic to parabolic).

\section{ACKNOWLEDGMENTS}

The author thanks Professor Ronald Grimmer for his valuable suggestions and comments.

\section{REFERENCES}

1. W. Desch and R. Grimmer, Propagation of singularities for integrodifferential equations, J. Differential Equations 65 (1986), 411-426.

2. W. Desch, R. Grimmer, and W. Schappacher, Propagation of singularities by solutions of second order integrodifferential equations, Volterra Integrodifferential Equations in Banach Spaces and Applications (G. Da Prato and M. Iannelli, eds.), Pitman Res. Notes Math. Ser., vol. 190, Longman Sci. Tech., Harlow, 1989, pp. 101-110.

3. (1984), 219-234.

4. W. Desch and W. Schappacher, A semigroup approach to integrodifferential equations in Banach space, J. Integral Equations Appl. 10 (1985), 99-110.

5. H. Fattorini, Second order linear differential equations in Banach spaces, North-Holland, Amsterdam, 1985, pp. 165-237.

6. J. Goldstein, Semigroups of linear operators and applications, Oxford Univ. Press, New York, 1985.

7. R. Grimmer and J. Liu, Integrodifferential equations with nondensely defined operators, Differential Equations with Applications in Biology, Physics, and Engineering (J. Goldstein, F. Kapple, and W. Schappacher, eds.), Marcel Dekker, New York, 1991, pp. 185-199.

8. _ـ Singular perturbations in viscoelasticity, Rocky Mountain J. Math. 24 (1994), 1-15.

9. J. Hale, Ordinary differential equations, Wiley-Interscience, New York, 1969, pp. 36-37.

10. J. Hale and G. Raugel, Upper semicontinuity of the attractor for a singularly perturbed hyperbolic equation, J. Differential Equations 73 (1988), 197-214.

11. R. MacCamy, An integro-differential equation with application in heat flow, Quart. Appl. Math. 35 (1977), 1-19.

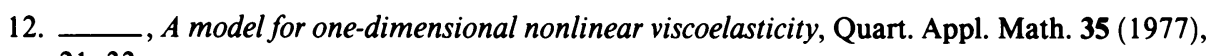
21-33.

13. D. Smith, Singular perturbation theory, Cambridge Univ. Press, Cambridge, 1985.

14. K. Tsuruta, Bounded linear operators satisfying second order integrodifferential equations in Banach space, J. Integral Equations 6 (1984), 231-268.

15. C. Travis and G. Webb, An abstract second order semi-linear Volterra integrodifferential equation, SIAM J. Math. Anal. 10 (1979), 412-424.

Department of Mathematics, James Madison University, Harrisonburg, Virginia 